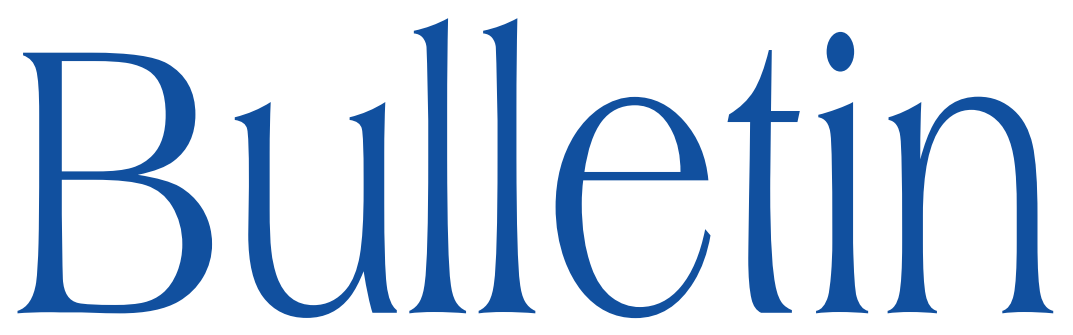

de la SOCIÉTÉ MATHÉMATIQUE DE FRANCE

\title{
ZÉRO-CYCLES DE DEGRÉ 1 SUR LES SOLIDES DE POONEN
}

Jean-Louis Colliot-Thélène

Tome 138

Fascicule 2

2010 


\title{
ZÉRO-CYCLES DE DEGRÉ 1 SUR LES SOLIDES DE POONEN
}

\author{
PAR JeAn-Louis Colliot-ThÉlène
}

\begin{abstract}
Résumé. - B. Poonen a récemment exhibé des exemples de variétés projectives et lisses de dimension 3 sur un corps de nombres qui n'ont pas de point rationnel et pour lesquelles il n'y a pas d'obstruction de Brauer-Manin après revêtement fini étale. Je montre que les variétés qu'il construit possèdent des zéro-cycles de degré 1 .
\end{abstract}

Abstract (Zero-cycles of degree 1 on Poonen threefolds). - B. Poonen recently produced smooth threefolds over a number field which do not have a rational point but have no Brauer-Manin obstruction even after descent to a finite étale cover. I show that the varieties he produces have zero-cycles of degree 1 .

\section{Introduction}

Soit $k$ un corps de nombres. Dans son article [10], B. Poonen construit des exemples de variétés projectives et lisses de dimension 3 sur $k$ qui ont les propriétés suivantes : elles ont des points dans tous les complétés de $k$, il n'y a pas d'obstruction de Brauer-Manin à l'existence d'un point rationnel, mieux, il n'y a pas d'obstruction de Brauer-Manin après descente par revêtements finis étales, et cependant les variétés ne possèdent pas de point $k$-rationnel. C. Demarche [5] vient de montrer que ceci implique qu'aucune obstruction de

Texte reçu le 22 septembre 2008, révisé le 16 février 2009 et accepté le 26 mai 2009

Jean-Louis Colliot-Thélène, C.N.R.S., UMR 8628, Mathématiques, Bâtiment 425, Université Paris-Sud, F-91405 Orsay, France • E-mail : jlct@math.u-psud.fr Classification mathématique par sujets (2000). — 14G05, 14G25, 11G35; 14J20, $14 \mathrm{~F} 22$. Mots clefs. - Points rationnels, zéro-cycles, principe de Hasse, obstruction de Brauer-Manin. 
descente sous un groupe algébrique ne saurait rendre compte de l'inexistence de point rationnel. La situation est donc tout à fait différente de celle de l'exemple historique de Skorobogatov ([12], [9]).

Dans [3] j'ai conjecturé que pour toute variété projective, lisse, géométriquement connexe sur un corps de nombres, l'obstruction de Brauer-Manin à l'existence d'un zéro-cycle de degré 1 est la seule obstruction.

Je montre ici que la conjecture vaut pour les variétés construites par Poonen : elles ont toutes un zéro-cycle de degré 1 . La question reste entière pour l'exemple de Skorobogatov.

$\mathrm{Au}$ paragraphe 1 je rappelle brièvement la construction des variétés. Au paragraphe 2 je donne une démonstration alternative du calcul du groupe de Brauer de ces variétés. Le résultat de ce calcul permet de montrer ([10]) qu'il n'y a pas d'obstruction de Brauer-Manin à l'existence d'un point rationnel, et a fortiori pas d'obstruction de Brauer-Manin à l'existence de zéro-cycles de degré 1 . Au paragraphe 3 , logiquement indépendant du précédent, j'établis l'existence de zéro-cycles de degré 1 . La méthode utilisée dans ce paragraphe est une variante pour les zéro-cycles d'une méthode connue dans le cas des points rationnels (voir les remarques 3.2 et 3.3 ci-dessous).

Cet article a été conçu à l'occasion d'exposés donnés en avril 2008 à l'université Emory (Atlanta, Géorgie).

\section{Les variétés}

Soit $k$ un corps de caractéristique nulle. Les variétés considérées dans [10] s'insèrent dans le diagramme suivant, que nous allons détailler.

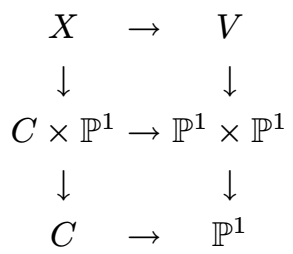

Dans ce diagramme, toutes les variétés sont des $k$-variétés projectives, lisses, géométriquement connexes. La variété $C$ est une courbe de genre quelconque. Dans [10], les courbes $C$ utilisées n'ont qu'un nombre fini de points rationnels, nous ne faisons pas cette restriction ici.

Les flèches verticales inférieures sont données par la projection sur le premier facteur. La variété $V$ est de dimension 3, elle est fibrée en coniques sur $\mathbb{P}^{1} \times \mathbb{P}^{1}$. Le lieu de dégénérescence de cette fibration (le lieu où la conique fibre est singulière) est une $k$-courbe projective, lisse, géométriquement intègre

TOME $138-2010-\mathrm{N}^{\circ} 2$ 
$Z_{1} \subset \mathbb{P}^{1} \times \mathbb{P}^{1}$. La projection sur le premier facteur $\mathbb{P}^{1} \times \mathbb{P}^{1} \rightarrow \mathbb{P}^{1}$ induit un revêtement ramifié $Z_{1} \rightarrow \mathbb{P}^{1}$, le lieu de ramification dans $\mathbb{P}^{1}$ ne contient pas le point $\infty \in \mathbb{P}^{1}(k)$.

Le morphisme $C \rightarrow \mathbb{P}^{1}$ est un revêtement ramifié, son lieu de ramification dans $\mathbb{P}^{1}$ ne rencontre pas le lieu de ramification de $Z_{1} \rightarrow \mathbb{P}^{1}$.

La partie verticale gauche du diagramme est obtenue par rétrotirette à partir de la partie verticale droite (produits fibrés via le morphisme $C \rightarrow \mathbb{P}^{1}$ ).

Pour tout point schématique $w \in \mathbb{P}^{1}$, de corps résiduel $k(w)$, la fibre $V_{w}$ est une surface géométriquement intègre sur $k(w)$ qui contient un ouvert affine d'équation

$$
y^{2}-a z^{2}=P_{w}(x)
$$

avec $a \in k \backslash k^{2}$ et $P_{w}(x) \in k(w)[x]$ un polynôme non nul, de degré 4 en la variable $x$, séparable pour $w$ non dans le lieu de ramification de $Z_{1} \rightarrow \mathbb{P}^{1}$. Sur un ouvert convenable de $\mathbb{P}^{1} \times \mathbb{P}^{1}, Z_{1}$ est défini par l'équation $P_{w}(x)=0$, la projection $Z_{1} \rightarrow \mathbb{P}^{1}$ étant donnée par $(w, x) \mapsto w$.

Le lieu de dégénérescence de la fibration en coniques $X \rightarrow C \times \mathbb{P}^{1}$ est l'image inverse $Z$ de $Z_{1}$ par $C \times \mathbb{P}^{1} \rightarrow \mathbb{P}^{1} \times \mathbb{P}^{1}$. Le polynôme $P_{w}(x)$ est choisi tel que que $Z \subset C \times \mathbb{P}^{1}$ est une $k$-courbe projective, lisse, géométriquement intègre ([10], Lemma 7.1).

Par ailleurs la description des fibres de l'application composée $V \rightarrow \mathbb{P}^{1} \times$ $\mathbb{P}^{1} \rightarrow \mathbb{P}^{1}$ (première projection) montre que l'on a :

Proposition 1.1. - Sous les hypothèses faites ci-dessus, pour tout point schématique $P$ de $C$, la fibre $X_{P} / k(P)$ du morphisme $X \rightarrow C$ au point $P$ est une $k(P)$-variété géométriquement intègre.

D'après [4, I, p. 43], [11, p. 208-209], [1, §3.2]), ceci a le corollaire suivant, qui sera utilisé au paragraphe 3 .

Corollaire 1.2. - Soient $k$ un corps de nombres et $X \rightarrow C$ comme cidessus. Il existe un ensemble fini $S$ de places de $k$ tel que, pour toute extension finie $L$ de $k$ et toute place $w$ de $L$ non située au-dessus d'une place de $S$, l'application $X\left(L_{w}\right) \rightarrow C\left(L_{w}\right)$ induite par $X \rightarrow C$ sur les points de $X$ à valeurs dans le complété $L_{w}$ soit surjective.

\section{Leur groupe de Brauer}

Le résultat suivant est établi dans [10] au moyen d'une étude de l'action du groupe de Galois sur le groupe de Picard géométrique de $X$. Nous proposons une démonstration un peu plus courte. La différence entre la démonstration de [10] et la présente démonstration est essentiellement la même que celle entre les propositions 7.1.1 et 7.1.2 de [13]. 
Proposition 2.1. - Sous les hypothèses ci-dessus, la flèche naturelle de groupes de Brauer $\operatorname{Br} C \rightarrow \operatorname{Br} X$ est un isomorphisme.

Démonstration. - Notons $B=C \times \mathbb{P}^{1}$. La fibration en coniques $X \rightarrow B$ est dégénérée en un seul point de codimension 1 de $B$, correspondant à la courbe géométriquement intègre $Z$.

Soit $\eta$ le point générique de $B$ et $X_{\eta}$ la fibre générique de $X \rightarrow B$. C'est une conique lisse.

De façon générale, étant donné un $k$-morphisme plat $X \rightarrow B$ de $k$-variétés lisses géométriquement intègres, on dispose d'un diagramme commutatif de suites exactes de groupes de cohomologie étale :

$$
\begin{array}{ccc}
0 \rightarrow \operatorname{Br} B & \rightarrow \operatorname{Br} k(B) \stackrel{\oplus_{b} \partial_{b}}{\longrightarrow} & \bigoplus_{b \in B^{(1)}} H^{1}(k(b), \mathbb{Q} / \mathbb{Z}) \\
\downarrow & \downarrow & \downarrow e_{x / b} \cdot \operatorname{Res}_{k(b) / k(x)} \\
0 \rightarrow \operatorname{Br} X \rightarrow \operatorname{Br} X_{\eta} \stackrel{\oplus_{x} \partial_{x}}{\longrightarrow} \bigoplus_{x \in B^{(1)}} \bigoplus_{x \in X^{(1)}, x \rightarrow b} H^{1}(k(x), \mathbb{Q} / \mathbb{Z}) .
\end{array}
$$

Dans ce diagramme, $b$ parcourt les points de codimension 1 de $B$, et $x$ parcourt les points de codimension 1 de $X$ qui ne sont pas situés sur $X_{\eta}$. Pour $x \in X$ d'image $b \in B$, on a l'inclusion des corps résiduels $k(b) \subset k(x)$. L'entier $e_{x / b}$ est l'indice de ramification de l'anneau de valuation discrète $\theta_{X, x}$ sur l'anneau de valuation discrète $\theta_{B, b}$, c'est-à-dire la valuation dans $\theta_{X, x}$ de l'image d'une uniformisante de $\theta_{B, b}$ par l'inclusion $\theta_{B, b} \subset \theta_{X, x}$. Les flèches $\partial$ sont les flèches de résidu.

On peut extraire un tel diagramme des exposés de Grothendieck sur le groupe de Brauer ([7], voir GB II, Cor. 1.10, GB III, Prop. 2.1, GB III, Thm. $6.1)$. Pour plus de détails, voir par exemple $[2, \S 3]$ et $[6$, Chapter 6$]$. La démonstration combine les suites de localisation, leur fonctorialité et le théorème de pureté pour le groupe de Brauer.

Dans la présente situation, la fibre générique est une conique sur $k(B)$. Soit $\beta \in \operatorname{Br} k(B)$ la classe de l'algèbre de quaternions associée à cette conique. Cette classe $\beta$ est non triviale, elle admet un unique résidu non trivial, au point générique de $Z$, et la classe correspondante est la classe de $a \in k^{*} / k^{* 2}=$ $H^{1}(k, \mathbb{Z} / 2) \subset H^{1}(k(Z), \mathbb{Q} / \mathbb{Z})$.

Pour la conique $X_{\eta}$ sur $k(B)$ sans point rationnel, on dispose de la suite exacte classique (cf. [12] p. 138)

$$
0 \rightarrow \mathbb{Z} / 2 \rightarrow \operatorname{Br} k(B) \rightarrow \operatorname{Br} X_{\eta} \rightarrow 0,
$$

où la flèche $\mathbb{Z} / 2 \rightarrow \operatorname{Br} k(B)$ envoie 1 sur la classe $\beta$. Le diagramme ci-dessus montre alors que l'application $\operatorname{Br} B \rightarrow \operatorname{Br} X$ est injective.

Soit $\alpha \in \operatorname{Br} X$. L'image de $\alpha$ dans $\operatorname{Br} X_{\eta}$ est l'image d'un élément $\gamma \in$ $\operatorname{Br} k(B)$, défini à addition près de $\beta$. 
Le lieu de dégénérescence de $X \rightarrow B$ est réduit à l'unique courbe $Z \subset B$. Ceci implique que pour $b \in B^{(1)}$ différent du point générique de $Z$, et $x$ l'unique point de $X^{(1)}$ au-dessus de $b$, qui définit une conique lisse donc géométriquement intègre sur le corps $k(b)$, la flèche $e_{x / b} \cdot \operatorname{Res}_{k(b) / k(x)}=\operatorname{Res}_{k(b) / k(x)}: H^{1}(k(b), \mathbb{Q} / \mathbb{Z}) \rightarrow H^{1}(k(x), \mathbb{Q} / \mathbb{Z})$ est une injection. Quant à la flèche $e_{x / b} \cdot \operatorname{Res}_{k(b) / k(x)}=\operatorname{Res}_{k(b) / k(x)}$ associée au point générique $b$ de $Z$ et à l'unique $x$ au-dessus de ce point, son noyau est le noyau de la flèche de restriction $H^{1}(k(Z), \mathbb{Q} / \mathbb{Z}) \rightarrow H^{1}(k(Z)(\sqrt{a}), \mathbb{Q} / \mathbb{Z})$. Ce noyau est d'ordre 2, engendré par la classe de $a$ dans $k^{*} / k^{* 2}=H^{1}(k, \mathbb{Z} / 2)$.

Du diagramme ci-dessus on conclut que les résidus de $\gamma$ aux points autres que le point générique de $Z$ sont nuls, et qu'au point générique de $Z$ le résidu est soit trivial soit égal au résidu de $\beta$. Quitte à remplacer éventuellement $\gamma$ par $\gamma+\beta$, ce qui est loisible, on voit que $\gamma \in \operatorname{Br} k(B)$ est dans l'image $\operatorname{de} \operatorname{Br} B$. Comme l'application $\operatorname{Br} X \rightarrow \operatorname{Br} X_{\eta}$ est injective, ceci achève de montrer que l'application $\operatorname{Br} B \rightarrow \operatorname{Br} X$ est un isomorphisme.

L'inclusion du point générique de $C$ dans $C$ définit un diagramme commutatif de morphismes de schémas réguliers intègres

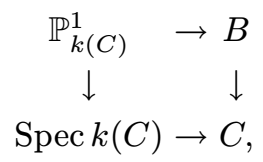

où la donnée d'un $k$-point de $\mathbb{P}_{k}^{1}$ induit des sections compatibles des flèches verticales. Ce diagramme induit un diagramme commutatif d'inclusions de groupes de Brauer

$$
\begin{array}{cc}
\operatorname{Br} B \subset \operatorname{Br} \mathbb{P}_{k(C)}^{1} \\
\uparrow & \uparrow \\
\operatorname{Br} C \subset \operatorname{Br} & k(C)
\end{array}
$$

pour lequel la donnée d'un $k$-point de $\mathbb{P}_{k}^{1}$ induit des rétractions compatibles des flèches verticales. Le groupe de Brauer de la droite projective sur un corps est égal à l'image du groupe de Brauer de ce corps. On voit ainsi que $\mathrm{Br} C \rightarrow \mathrm{Br} B$ est un isomorphisme.

Ainsi la flèche composée $\mathrm{Br} C \rightarrow \mathrm{Br} B \rightarrow \mathrm{Br} X$ est un isomorphisme.

\section{Existence de zéro-cycles de degré 1}

ThÉORÈme 3.1. - Soit $k$ un corps de nombres. Soient $V \rightarrow \mathbb{P}^{1} \times \mathbb{P}^{1}, C \rightarrow \mathbb{P}^{1}$ et $X \rightarrow C \times \mathbb{P}^{1}$ comme ci-dessus. Faisons les deux hypothèses :

(i) la fibre (lisse) $V_{\infty}$ de la fibration composée $V \rightarrow \mathbb{P}^{1} \times \mathbb{P}^{1} \rightarrow \mathbb{P}^{1}$ (première projection) a des points dans tous les complétés de $k$;

(ii) il existe un $k$-point $P$ de $C$ dont l'image par $C \rightarrow \mathbb{P}^{1}$ est le point $\infty$. 
Alors pour tout entier $n \geq 2 g+1$, où $g$ désigne le genre de $C$, la $k$-variété $X$ possède un point dans un corps extension de $k$ de degré $n$. En particulier $X$ possède un zéro-cycle de degré 1.

Démonstration. - Soit $n$ comme dans l'énoncé. Le système linéaire associé au diviseur $n P$ de $C$ est alors très ample. Soit $C \hookrightarrow \mathbb{P}^{N}$ le plongement associé, et soit $\check{\mathbb{P}}^{N}$ l'espace projectif dual de $\mathbb{P}^{N}$.

La variété d'incidence $W \subset \mathbb{P}^{N} \times \check{\mathbb{P}}^{N}$ définie par l'annulation de la forme bihomogène $\sum_{i=0}^{N} X_{i} Y_{i}$ est un diviseur très ample. La projection $W \rightarrow \mathbb{P}^{N}$ définit un fibré (lisse) en espaces projectifs $\mathbb{P}^{N-1}$.

Soit $W_{Z} \subset Z \times \check{\mathbb{P}}^{N}$ l'image inverse de la correspondance d'incidence via le morphisme composé

$$
Z \times \check{\mathbb{P}}^{N} \rightarrow C \times \check{\mathbb{P}}^{N} \rightarrow \mathbb{P}^{N} \times \check{\mathbb{P}}^{N}
$$

C'est un fibré (lisse) en espaces projectifs $\mathbb{P}^{N-1}$ au-dessus de la courbe $Z$, qui est projective, lisse et géométriquement intègre. Ainsi $W_{Z}$ est projectif, lisse et géométriquement intègre.

Le même argument montre que l'image inverse $W_{C} \subset C \times \check{\mathbb{P}}^{N}$ de la correspondance d'incidence est une $k$-variété projective, lisse, géométriquement intègre.

Les morphismes naturels $W_{Z} \rightarrow W_{C} \rightarrow \check{\mathbb{P}}^{N}$ sont des morphismes propres dominants et finis - ils sont quasi-finis car la courbe $C$ engendre $\mathbb{P}^{N}$ projectivement. Le morphisme $W_{Z} \rightarrow W_{C}$ est de degré 4 , le morphisme $W_{C} \rightarrow \check{\mathbb{P}}^{N}$ est de degré $n$.

Etant donné un $k$-point $h \in \check{\mathbb{P}}^{N}(k)$ correspondant à un hyperplan $H$ de $\check{\mathbb{P}}^{N}$, la fibre de $W_{C} \rightarrow \check{\mathbb{P}}^{N}$ en le point $h$ est le $k$-schéma $C \cap H \subset C$.

Pour toute place $v$ de $k$, la fibre (lisse) $X_{P}$ du morphisme $X \rightarrow C$ au-dessus du $k$-point $P$ possède un $k_{v}$-point lisse. Ceci implique que pour toute extension finie $L / k_{v}$, l'image de l'application induite $X(L) \rightarrow C(L)$ contient un voisinage ouvert $U_{L}$ de $P$ pour la topologie $v$-adique sur $C(L)$.

Il existe un hyperplan $H_{0} \in \check{\mathbb{P}}^{N}(k)$ qui découpe exactement le diviseur $n P$ sur $C$. Soit $v$ une place de $k$. Tout hyperplan $H \in \check{\mathbb{P}}^{N}(k)$ qui est suffisamment proche de $H_{0}$ pour la topologie $v$-adique découpe sur la courbe $C$ une somme de points fermés qui, lorsque l'on passe de $k$ à un complété $k_{v}$, donnent des points sur diverses extensions finies $L$ de $k_{v}$ de degré au plus $n$, points qui sont dans $U_{L}$. Rappelons qu'un tel corps local $k_{v}$ (de caractéristique nulle) n'admet qu'un nombre fini d'extensions $L / k_{v}$ de degré au plus $n$.

Appliquons le théorème d'irréductibilité de Hilbert avec approximation faible (cf. [8], Prop. 3.2.1) au revêtement fini de variétés géométriquement intègres 
$W_{Z} \rightarrow \check{\mathbb{P}}^{N}$, composé de $W_{Z} \rightarrow W_{C} \rightarrow \check{\mathbb{P}}^{N}$ et à un ensemble fini $S$ de places de $k$ donné par le corollaire 1.2.

On trouve ainsi un point $h \in \check{\mathbb{P}}^{N}(k)$ définissant un hyperplan $H$ de $\mathbb{P}^{N}$ tel que les propriétés suivantes soient satisfaites.

(a) La fibre de $W_{Z} \rightarrow \check{\mathbb{P}}^{N}$ en $h$ est intègre, et donc :

(b) La fibre de $W_{C} \rightarrow \check{\mathbb{P}}^{N}$ en $h$, qui est le schéma $H \cap C \subset C$, définit un point fermé $M \in C$ de degré $n$ sur $k$, et la fibre du revêtement $Z \rightarrow C$ en $M$ est intègre, de degré 4 sur le corps résiduel $k(M)$.

Ceci implique :

(c) La fibre $X_{M}$ de $X \rightarrow C$ au-dessus du point $M$ est une surface projective, lisse et géométriquement intègre sur le corps $k(M)$, surface qui admet un modèle affine d'équation $y^{2}-a z^{2}=P_{M}(x)$ avec $P_{M}(t) \in k(M)[t]$ un polynôme irréductible de degré 4.

L'application du théorème d'irréductibilité avec approximation permet de choisir l'hyperplan $H$ très proche de $H_{0}$, pour la topologie $v$-adique, aux places $v \in S$, de façon que :

(d) Pour tout corps $L$ complété de $k(M)$ en une place $w$ au-dessus d'une place $v \in S$, le point $M$ appartient à $U_{L}$.

D'après ce que l'on a vu plus haut, ceci assure que la $k(M)$-surface $X_{M}$ possède des points dans tous les complétés de $k(M)$ aux places au-dessus d'une place de $S$. Par ailleurs, le choix de $S$ (voir le corollaire 1.2) assure que la $k(M)$-surface $X_{M}$ a des points dans tous les complétés de $k(M)$ aux places non situées au-dessus d'une place de $S$.

En conclusion, la $k(M)$-surface lisse $X_{M}$ est une surface de Châtelet qui possède des points dans tous les complétés de $k(M)$ et qui admet une équation affine $y^{2}-a z^{2}=P(x)$ avec $P(x) \in k(M)[x]$ irréductible de degré 4. L'un des principaux résultats de [4], le théorème 8.11, assure que le principe de Hasse vaut pour une telle surface. Ainsi $X_{M}(k(M)) \neq \varnothing$, a fortiori $X(k(M)) \neq \varnothing$ : la $k$-variété $X$ possède un point dans l'extension $k(M) / k$, qui est de degré $n$.

Remarque 3.2. Supposons $C=\mathbb{P}^{1}$. Dans ce cas on peut prendre $n=1$, et on obtient $X(k) \neq \varnothing$. On a mieux. Comme les surfaces de Châtelet d'équation affine $y^{2}-a z^{2}=P(x)$ avec $P(x)$ irréductible satisfont l'approximation faible ([4, Thm. 8.11]), une variante immédiate de l'argument établit l'approximation faible pour $X$ : l'ensemble $X(k)$ est dense dans le produit des $X\left(k_{v}\right)$. L'argument est un cas particulier d'un théorème général sur des familles $X \rightarrow \mathbb{P}^{1}$ de variétés dont toutes les fibres sont géométriquement intègres (Harari, [8], Thm. 4.2.1). 
On peut d'ailleurs voir la démonstration ci-dessus comme une version de ce théorème dans le cadre des zéro-cycles, et ce au-dessus d'une courbe $C$ quelconque.

Remarque 3.3. Dans [3], outre la conjecture sur l'existence de zéro-cycles de degré 1, j'ai proposé une conjecture sur l'application diagonale de groupes de Chow de zéro-cycles $\mathrm{CH}_{0}(X) \rightarrow \prod_{v} \mathrm{CH}_{0}\left(X_{v}\right)$. Dans une série d'articles (voir la bibliographie de [3]), des versions de cette conjecture ont été établies pour des variétés $X$ fibrées en variétés de Severi-Brauer au-dessus d'une courbe $C$, sans supposer que toutes les fibres de $X \rightarrow C$ sont géométriquement intègres. Les arguments sont beaucoup plus sophistiqués que ceux du présent article.

Il serait intéressant de voir si l'on peut pousser les arguments ci-dessus et établir la conjecture pour les familles $X \rightarrow C$ de surfaces de Châtelet considérées ici, qui ont la propriété que toutes les fibres de $X \rightarrow C$ sont géométriquement intègres.

Il serait sans doute très difficile de relâcher cette dernière condition.

\section{BIBLIOGRAPHIE}

[1] J.-L. Colliot-ThÉlÈne - «L'arithmétique des variétés rationnelles », Ann. Fac. Sci. Toulouse Math. 1 (1992), p. 295-336.

[2] _ _ Birational invariants, purity and the Gersten conjecture », in $K$-theory and algebraic geometry : connections with quadratic forms and division algebras (Santa Barbara, CA, 1992), Proc. Sympos. Pure Math., vol. 58, Amer. Math. Soc., 1995, p. 1-64.

[3] _ _ « Conjectures de type local-global sur l'image des groupes de Chow dans la cohomologie étale », in Algebraic K-theory (Seattle, WA, 1997), Proc. Sympos. Pure Math., vol. 67, Amer. Math. Soc., 1999, p. 1-12.

[4] J.-L. Colliot-Thélène, J.-J. Sansuc \& P. Swinnerton-Dyer - «Intersections of two quadrics and Châtelet surfaces », J. reine angew. Math. 373 (1987), p. 37-107; II, ibid. 374 (1987), p. 72-168.

[5] C. Demarche - «Obstruction de descente et obstruction de BrauerManin », Algebra and Number Theory 3 (2008), p. 237-254.

[6] P. Gille \& T. Szamuely - Central simple algebras and Galois cohomology, Cambridge Studies in Advanced Math., vol. 101, Cambridge Univ. Press, 2006.

[7] A. Grothendieck - «Le groupe de Brauer, I, II, III », in Dix exposés sur la cohomologie des schémas, Advanced Studies in Pure Mathematics, Masson, 1968. 
[8] D. HARARI - «Méthode des fibrations et obstruction de Manin », Duke Math. J. 75 (1994), p. 221-260.

[9] D. Harari \& A. N. Skorobogatov - « Non-abelian cohomology and rational points », Compositio Math. 130 (2002), p. 241-273.

[10] B. Poonen - «Insufficiency of the Brauer-Manin obstruction applied to étale covers », prépublication arXiv:0806.1312 à paraître dans Annals of Math.

[11] A. N. Skorobogatov - «On the fibration method for proving the Hasse principle and weak approximation », in Séminaire de Théorie des Nombres, Paris 1988-1989, Progr. Math., vol. 91, Birkhäuser, 1990, p. 205-219.

[12] _ « Beyond the Manin obstruction », Invent. Math. 135 (1999), p. $399-424$.

[13] _ Torsors and rational points, Cambridge Tracts in Mathematics, vol. 144, Cambridge Univ. Press, 2001. 